\title{
Effect of Ionic Surfactant on the Self-assembly of Triblock Copolymer
}

\author{
D. Ray ${ }^{1 *}$, J. Kohlbrecher ${ }^{2}$ and V. K. Aswal ${ }^{1}$ \\ ${ }^{1}$ Solid State Physics Division, Bhabha Atomic Research Centre, Mumbai 400 085, India \\ ${ }^{2}$ Laboratory for Neutron Scattering, Paul Scherrer Institut, CH-5232 PSI Villigen, Switzerland \\ ${ }^{\star}$ E-mail:debes@barc.gov.in
}

\begin{abstract}
The effect of anionic surfactant sodium dodecyl sulfate (SDS) on the self-assembly of PEO-PPO-PEO triblock copolymer in aqueous solution has been studied using small-angle neutron scattering (SANS). The measurements have been carried out for fixed concentrations (1 wt \%) of block copolymer and surfactant, and at varying temperatures between 20 to $80^{\circ} \mathrm{C}$. Micelles of pure ionic surfactants show little change in their sizes with increasing temperature, whereas structures of the non-ionic micelles increase significantly (sphere-to-rod transition for some block copolymers). Contrast variation SANS measurement suggests addition of SDS to aqueous copolymer solutions leads to the formation of mixed micelles. Interestingly, the self-assembly of block copolymer is found to get completely arrested by the ionic surfactant in the mixed micelles which gives a control over the block copolymer phase behavior.
\end{abstract}

Keywords: Block copolymer, surfactant, small-angle neutron scattering

PACS: 82.35.Jk, 82.70.Uv, 61.05.fm

\section{INTRODUCTION}

Due to their rich structural polymorphism, Pluronic poly(ethylene oxide)-poly(propylene oxidepoly(ethylene oxide) PEO-PPO-PEO triblock copolymers are an interesting class of amphiphilic soft materials, and used extensively in several applications like nanoparticle synthesis, drug delivery, polymer gel, templating agents etc [1-3]. PEO-PPO-PEO triblock copolymers comprise of two dissimilar moieties, hydrophilic PEO block and hydrophobic PPO block, covalently linked within the same molecule. In the aqueous medium, strongly temperature-dependence and differential solubility characteristics of the PEO and PPO blocks play an important role in determining the self-assembly characteristics of these copolymers.

At low temperature, the PPO and PEO blocks are aqueous soluble and the molecules of both of these copolymers remain singly dissolved as unimers. Above the critical micellar temperature (CMT), they start forming micelles comprising a hydrophobic core containing the PPO blocks and a hydrated corona containing the PEO blocks. Since the solubility of the PPO block decreases with an increase in temperature above the CMT, the micelles undergo restructuring to increase their aggregation number and core size and to decrease their degree of hydration. Block copolymers self-assemble in different manner with the variation in temperature and form various self-assembled nanostructures [1].
The majority of applications relating to block copolymer use them in the presence of various cosolvents or surfactants, and therefore, the influence of surfactants in general and ionic surfactant in particular on the association behavior of block copolymers is very crucial. The combination of nonionic block copolymers and ionic surfactants is being utilized for many possibilities in designing new polymeric materials, catalyst, polymer membrane, metal ion removal etc. In view of this, it is important to look into the effect of these kind of mixed systems, their interactions and resultant structures. In the present work, we have examined the effect of anionic surfactant sodium dodecyl sulfate (SDS) on the temperature-dependent self-assembly of triblock copolymer P85 on as in their mixed system in aqueous solution using small-angle neutron scattering (SANS). Unimers of P85 self-assembles to form spherical micelles above CMT and further above $75^{\circ} \mathrm{C}$ show sphere-to-rod micellar shape transition. On the purview of this, it would be interesting to see the effect of ionic surfactant on the self-assembly of P85. For this we have performed the experiments on the individual components as well as their mixed system at three different temperatures, 20,40 and $80^{\circ} \mathrm{C}$.

\section{EXPERIMENTAL}

Pluronic block copolymer P85 $\left(\mathrm{EO}_{26} \mathrm{PO}_{39} \mathrm{EO}_{26}\right.$, M.W. $=4600)$ and surfactant SDS $(\mathrm{M} . \mathrm{W} .=288.372)$ were purchased from BASF Corp. and Sigma Aldrich, 
respectively. $99.9 \%$ pure $\mathrm{D}_{2} \mathrm{O}$ were used for sample preparation. SANS experiments were performed at SANS diffractometers at Dhruva Reactor, BARC [4] and PSI, Switzerland [5]. The instrument covers a $Q$ range of $0.005-0.3 \AA^{-1}$. The data were corrected and normalized to absolute scale using the standard procedure.

\section{Small-angle Neutron Scattering Analysis}

In SANS, one measures the differential scattering cross-section $(d \Sigma / d \Omega)$ per unit volume as a function of wave vector transfer $Q$, which for a system of monodisperse particles in a medium can be expressed as $\left(\frac{d \Sigma}{d \Omega}\right)(Q)=n V^{2}\left(\rho_{p}-\rho_{s}\right)^{2} P(Q) S(Q)+B$,

where $n$ denotes the number density of particles, $\rho_{p}$ and $\rho_{s}$ are, respectively, the scattering length densities of particle and solvent and $V$ is the volume of the particle. $P(Q)$ is the intraparticle structure factor and $S(Q)$ is the interparticle structure factor. $B$ is a constant term representing incoherent background, which is mainly due to the hydrogen present in the sample.

Intraparticle structure factor $P(Q)$ is decided by the shape and size of the particle and is the square of single-particle form factor $F(Q)$ as determined by

$$
P(Q)=\left\langle|F(Q)|^{2}\right\rangle \text {. }
$$

For a spherical particle of radius $R, F(Q)$ is given by

$$
F(Q)=3\left[\frac{\sin (Q R)-Q R \cos (Q R)}{(Q R)^{3}}\right] .
$$

The expression for form factor of prolate ellipsoidal particle with semi-major axis $a$ and semi-minor axis $b=c$ is given by

$P(Q)=\int_{0}^{1} F(Q, \mu)^{2} d \mu$, where

$F(Q, x)=\left[\frac{3(\sin x-x \cos x)}{x^{3}}\right]^{2}, \quad x=Q\left[a^{2} \mu^{2}+b^{2}\left(1-\mu^{2}\right)\right]^{\frac{1}{2}}$

and $\mu$ is the cosine of the angle between the direction of major axis and wave vector transfer $Q$.

$S(Q)$ describes the interaction between the particles present in the system and it is the Fourier transform of the pair correlation function for the mass centers of the particles. For dilute systems, $S(Q) \sim 1$.

In the case of an interacting isotropic system,

$$
S(Q)=1+4 \pi n \int[g(r)-1] \frac{\sin Q r}{Q r} r^{2} d r
$$

where $\mathrm{g}(\mathrm{r})$ is the radial distribution function. It has been calculated for ionic micelles as derived by Hayter and Penfold from the Ornstein-Zernike equation and using the mean spherical approximation for charged macroions [6].

The data have been analyzed by comparing the scattering from different models to the experimental data. The fitted parameters in the analysis were optimized using nonlinear least-square fitting program to the model scattering.

\section{RESULTS AND DISCUSSION}

Figure 1 shows the SANS data of 1 wt $\%$ block copolymer P85 at varying temperatures. The scattering intensity for P85 is too low at $20{ }^{\circ} \mathrm{C}$ (below CMT, $29.5^{\circ} \mathrm{C}$ ) as the solution mainly consists of unimers. The increase in the scattered intensity with the increase in the temperature (at $40^{\circ} \mathrm{C}$ ) seen is due to the enhanced micellization of block copolymers in the solution. At temperatures higher than the CMT of the respective block copolymer, the micelles are in thermodynamic equilibrium with the unimers. At further higher temperature $\left(80^{\circ} \mathrm{C}\right)$ scattering shows a linear $Q$-dependence on log-log scale in low- $Q$ region, suggesting temperature-induced sphere-to-rod-like transition for P85 micelles. It is the decreased hydration of the head group that increases the size of non-ionic micelles with an increase in temperature. The micellar parameters of P85 obtained from data analyses are given in Table 1. Inset of Figure 1 shows the SANS data of $1 \mathrm{wt} \%$ SDS at different temperatures. A strong correlation peak appears in the data due to the charged repulsion between SDS micelles. It is observed that upon increasing the temperature, ionic micelles show a nominal decrease in the size. This is why anionic SDS has been chosen to see its role on the block copolymer self-assembly.

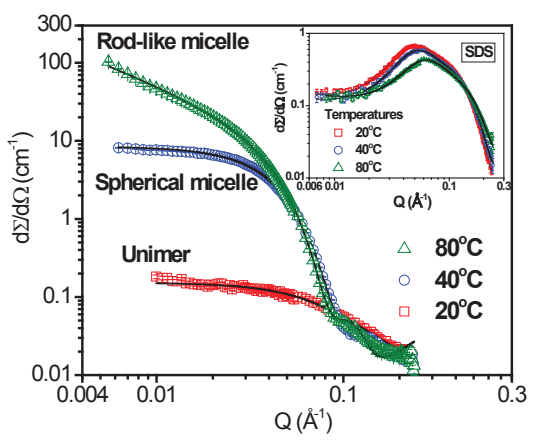

FIGURE 1. SANS data of $1 \mathrm{wt} \% \mathrm{P} 85$ and $1 \mathrm{wt} \%$ SDS (inset) with varying temperature.

TABLE 1. Fitted structural parameters of self-assembled micellar structures of $1 \mathrm{wt} \% \mathrm{P} 85$ with varying temperature.

\begin{tabular}{cccc}
\hline Block & Temp. $\left({ }^{\mathbf{O}} \mathbf{C}\right)$ & \multicolumn{2}{c}{ Micellar Parameters } \\
\cline { 2 - 4 } Copolymer & & Shape & Dimensions \\
\hline \multirow{2}{*}{$\begin{array}{c}\text { Pt } \% \\
\text { P85 }\end{array}$} & $\begin{array}{c}\text { Gaussian } \\
\text { chain }\end{array}$ & $\begin{array}{c}\text { Radius of } \\
\text { gyration }=12 \AA\end{array}$ \\
\cline { 2 - 4 } & 80 & Spherical & $\mathrm{R}=36.2 \AA$ \\
\hline
\end{tabular}


Figure 2 shows the SANS data of mixed system of $1 \mathrm{wt} \% \mathrm{P} 85$ and $1 \mathrm{wt} \%$ SDS at three different temperatures, i.e. $20^{\circ} \mathrm{C}, 40^{\circ} \mathrm{C}$ and $80^{\circ} \mathrm{C}$. Surprisingly, no signature of the self-assembly induced drastic shape change of block copolymer micelles (unimer-tospherical micelles-to-rodlike micelles) has been reflected on the scattering data of the mixed system. The correlation peak in the mixed micelles confirms the interaction among these micelles which is attributed to their ionic nature. The data at varying temperatures are almost overlapping, which indicates no structural change. The micellar parameters obtained from the data analyses for P85-SDS mixed system with increasing temperature are given in Table 2 . Debye length $\left(\lambda_{D}\right)$, the distance over which the charged surface of the particle is shielded from the bulk, has also been calculated from structure factor analysis to study the mixed micellar interaction.

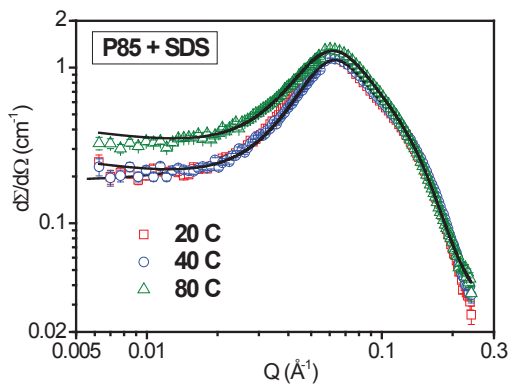

FIGURE 2. SANS data of mixed system of $1 \mathrm{wt} \% \mathrm{P} 85$ and $1 \mathrm{wt} \%$ SDS at temperatures $20^{\circ} \mathrm{C}, 40^{\circ} \mathrm{C}$ and $80^{\circ} \mathrm{C}$.

TABLE 2. Fitted structural parameters of self-assembled micellar structures of $1 \mathrm{wt} \% \mathrm{P} 85$ with varying temperature.

\begin{tabular}{ccccc}
\hline System & $\begin{array}{c}\text { Temp. } \\
\left({ }^{\mathbf{0}} \mathbf{C}\right)\end{array}$ & $\begin{array}{c}\text { Semi- } \\
\text { major } \\
\text { axis }(\AA)\end{array}$ & $\begin{array}{c}\text { Semi- } \\
\text { minor } \\
\text { axis }(\AA)\end{array}$ & $\begin{array}{c}\text { Fractional } \\
\text { charge } \\
(\mathbf{e s u})\end{array}$ \\
\hline $\begin{array}{c}1 \mathrm{wt} \% \\
\mathrm{P} 85 \\
+\end{array}$ & 20 & 26.4 & 16.7 & 0.25 \\
\cline { 2 - 5 } $\begin{array}{c}\mathrm{wt} \% \\
\mathrm{SDS}\end{array}$ & 40 & 26.2 & 16.7 & 0.25 \\
\cline { 2 - 5 } & 80 & 26.7 & 16.7 & 0.23 \\
\hline
\end{tabular}

To look into the mixed system more precisely contrast-matching SANS experiments have been carried out. Figure 3 shows the SANS data of mixed system of $1 \mathrm{wt} \% \mathrm{P} 85$ and $1 \mathrm{wt} \% \mathrm{SDS}$ at $40^{\circ} \mathrm{C}$. The two data sets are shown without and with SDS contrast matched with the solvent. It has been done by using the hydrogenous (h-SDS) and deuterated SDS (d-SDS) surfactant in the mixed system. It is observed that the scattering cross-section decreases as the SDS surfactant is contrast matched with the solvent as the contrast factor of mixed micelle is decreased. The parallel data sets without and with the deuterated surfactant suggests homogeneous mixing of the two components in the mixed micelles [7]. This is because only in the case of mixed micelles, the structures of the micelles remain same whereas its contrast changes. Hence it is clear that non-ionic P85 forms ionic mixed micelles in presence of ionic SDS. The self-assembly of block copolymer is found to get completely arrested by the ionic surfactant in the mixed micelles.

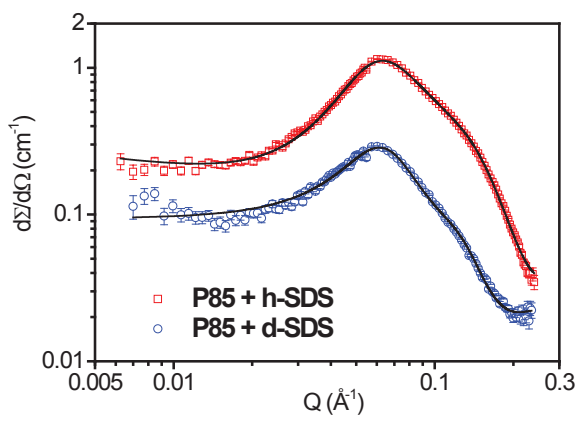

FIGURE 3. SANS data of mixed system of $1 \mathrm{wt} \% \mathrm{P} 85$ and $1 \mathrm{wt} \%$ SDS without and with deuterated surfactant.

\section{CONCLUSIONS}

The role of anionic surfactant SDS on the temperature-dependent self-assembly of non-ionic block copolymer P85 has been studied by SANS. P85 micelles form mixed micelles with SDS micelles independent of temperature. These mixed micelles with the head group of SDS hidden in the corona are ionic in nature, and restrict the self-assembled growth of P85. Thus the presence of ionic surfactant gives a control over the block copolymer self-assembly and subsequently their phase behavior.

\section{REFERENCES}

1. F. H. Schacher, P. A. Rupar and I. Manners, Angew. Chem. Int. Ed. 51, 7898-7921 (2012).

2. D. Ray, V. K. Aswal and D. Srivastava, J. Nanosci. Nanotechnol. 11, 1905-1913 (2011).

3. D. Ray, V. K. Aswal and J. Kohlbrecher, Langmuir 27, 4048-4056 (2011).

4. V. K. Aswal and P. S. Goyal, Curr. Sci. 79, 947-953 (2000).

5. J. Kohlbrecher and W. Wagner, J. Appl. Cryst. 33, 804$806(2000)$.

6. J. B. Hayter and J. Penfold, Colloid Polym. Sci. 261, 1022-1030 (1983).

7. R. Ganguly, V. K. Aswal, P. A. Hassan, I. K. Gopalakrishnan and S. K. Kulshreshtha, J. Phys. Chem. B 110, 9843-9849 (2006). 